\title{
Hombres en profesiones de cuidado tradicionalmente feminizadas
}

\section{Men in traditionally feminized care professions}

\author{
Juan Guillermo Figueroa y Tania Hernández \\ Centro de Estudios Demográficos, Urbanos y Ambientales \\ de El Colegio de México, México
}

\section{Resumen}

Este texto busca documentar las experiencias de algunos varones en profesiones feminizadas. Se plantean tres ejes de análisis: a) la llegada, expectativas y salida de las profesiones feminizadas, b) ventajas y desventajas dentro de la profesión y c) experiencias de cuidado más allá de su profesión. Se recurre a entrevistas a profundidad a siete varones. Destaca que los varones llegan a las profesiones por una mezcla de expectativas económicas y de desarrollo personal. Reportan discriminación de mujeres adultas mayores (entre los enfermeros) y de otros varones en su profesión (entre los profesores). En el ámbito doméstico llevan a cabo actividades de cuidado de sus hijos y padres. Se concluye con una ruta de temas a profundizar.

Palabras clave: Profesiones feminizadas, cuidado, enfermeros, maestros.

\section{Abstract}

This paper aims to research on the experiences of some men in feminized professions. We developed three levels of analysis: a) the arrival, expectations and exit of the males from these professions, b) advantages and disadvantages within the profession, and c) their experiences of care beyond their profession. A qualitative method was used through in-depth interviews with seven men. Findings suggest that men come to the professions for a mix of economic expectations and personal development. Males reported being victims of discrimination by older adult women (the nurses) and other males in their profession (the teachers). In the domestic sphere, carry out care activities for their children and parents. We conclude with a route of topics to go in depth.

Key words: Feminized professions, care activities, nurses, teachers. 


\section{INTRODUCCIÓN}

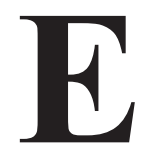

ste artículo presenta los hallazgos de una investigación realizada en 2009 con hombres mexicanos en profesiones de cuidado, asociadas tradicionalmente con alta participación femenina. De acuerdo con Buquet (2006), se entiende por profesiones feminizadas aquellas en las que más de la mitad de sus participantes son mujeres. ${ }^{1}$ A partir de los datos de la Encuesta Nacional de Ocupación y Empleo (ENOE) de 2014 y 2015, existían en México 475 mil personas dedicadas a la enfermería, de las cuales 85 por ciento son mujeres y 15 por ciento son hombres. Por otro lado, existen un millón 203 mil maestros de educación básica, ${ }^{2}$ de los cuales 67.9 por ciento son mujeres y 31.8 por ciento son hombres (INEGI, 2014 y 2015). Con base en estas cifras se puede hablar de dos profesiones con alta participación femenina y que concentran nuestro interés en este texto. Si bien los datos estadísticos son posteriores al desarrollo de la investigación que alimenta este texto, la idea es mostrar la permanencia actual de la temática en estudio.

La pregunta que orienta esta investigación es ¿cuáles son las experiencias de los hombres en profesiones feminizadas? De esta, se generaron tres más específicas: ¿cómo llegan los varones a las profesiones feminizadas?, ¿qué ventajas y desventajas presentan los hombres en trabajos feminizados? y ¿cómo son los arreglos de cuidado en casa, de los hombres en trabajos feminizados? Se construye un diálogo con estos cuestionamientos, con el fin de formular hipótesis alrededor de la experiencia de los varones en ámbitos específicos de cuidado, los cuales se asumen tradicionalmente como competencia de las mujeres (Arango y Molinier, 2011). Esto permite seguir construyendo conocimiento en este ámbito, a partir de cuestionar estereotipos de género y de problematizar algunos supuestos. La intención del texto es más de orden reflexivo, visitando los relatos de una población menos estudiada en labores de cuidado.

El texto se estructura en tres secciones, en la primera se recuperan algunos trabajos precedentes que han estudiado a los hombres en profesiones feminizadas. En la segunda se presenta la metodología utilizada para la

\footnotetext{
${ }^{1}$ Estudios similares se condujeron en Brasil, Chile, India y Sudáfrica como parte del proyecto de investigación The Men and Gender Equality Policy Project coordinado por el Instituto Promundo y el International Center for Research on Women (Barker et al., 2012; Figueroa y Flores, 2012). Este proyecto recibió financiamiento de la Fundación MacArthur.

${ }^{2}$ La educación básica incluye preescolar, primaria y secundaria.
} 
presente investigación, así como el contenido general de las entrevistas realizadas a los hombres en profesiones feminizadas. En la tercera sección, se presentan los hallazgos de las entrevistas y algunas reflexiones y propuestas de investigación con base en dichos hallazgos.

\section{ESTUDIOS PREVIOS DE HOMBRES EN PROFESIONES FEMINIZADAS}

La profesión que ha sido objeto de análisis de la mayoría de estas investigaciones, sobre cuidado por parte de hombres, es la enfermería, es decir, la participación de los hombres en este oficio (Williams, 1995b; Evans, 1997; Kleinman, 2004; Romem y Anson, 2005; Meadus, 2000; Molinier, 2011; Lara, 2012). Otras investigaciones se han centrado en hombres bibliotecarios (Piper y Collamer, 2001) y en vendedores ambulantes (Agadjanian, 2002). Existen otros estudios que incorporan diversas profesiones. Entre estos se encuentran los trabajos de Lupton (2000 y 2006), que incluye en su estudio a administradores de recursos humanos, maestros de primaria y bibliotecarios, y Simpson (2004), que incluye bibliotecarios, auxiliares de vuelo, enfermeros y maestros de primaria.

Los estudios de hombres en profesiones feminizadas se han enfocado a tres aspectos principalmente: la entrada de los hombres a la profesión, las desventajas de los hombres en dicho ámbito y las disparidades de género a través de comparaciones entre hombres y mujeres. Estudios sobre enfermeros (Romem y Anson, 2005) advierten que los hombres entraron a la profesión debido a una exposición temprana a la misma, a través de experiencias de familiares o amistades. La retribución económica no fue un motivo para la elección profesional, pero sí lo fueron los beneficios asociados a ásta, como el brindar cuidado y la estabilidad laboral.

Sobre los retos que presentan los hombres en profesiones feminizadas, Lupton advierte tres áreas problemáticas: i) el reto en el espacio de trabajo como una arena para ejercer y regenerar su masculinidad, ii) el miedo de ser feminizado por la exposición a las mujeres (feminización) y iii) la amenaza de ser estigmatizados como afeminado u homosexual por la asociación con mujeres o por hacer "trabajo de mujeres" (estigmatización) (Lupton, 2000: S38).

Sobre el primer reto, Lupton (2000) observa que los varones pueden percibir que su habilidad para atraer pareja se ve comprometida por la percepción de bajo estatus en su trabajo. En el espacio de trabajo evitan las conversaciones "femeninas", son menos rudos, en términos de lenguaje utilizado y se sienten más cómodos trabajando con mujeres jóvenes que con mujeres de mayor edad. Por otro lado, los hombres son objeto de bro- 
mas por parte de las mujeres. Sobre este aspecto ellos observan que, si fueran en el sentido contrario, éstas serían consideradas conductas inapropiadas e incluso podrían ser catalogadas como acoso sexual.

Sobre el segundo reto, Lupton (2000) destaca que los hombres presentan miedo de feminización por la exposición a las mujeres en tres áreas: i) convertirse en una más de "las chicas", ii) ser tratado como mujer, y iii) feminizarse por trabajar con mujeres. Los hombres generan una diversidad de respuestas a estos retos, como ejemplo, se encuentra el acentuar las características que ellos consideran diferenciadoras entre hombres y mujeres.

El tercer reto que Lupton (2000) observa es el miedo de los hombres a ser estigmatizados como afeminados y homosexuales. Esto es consistente con los hallazgos de Evans (2002), quien observa que los enfermeros perciben un cuestionamiento a su identidad, por el estereotipo de que los hombres son considerados como agresores sexuales, o bien como desviados sexuales, en el caso de hombres homosexuales. Mientras que el acercamiento y contacto físico de la mujer con la persona que se cuida podría ser considerado natural, como una extensión a su rol tradicional de cuidadora, de acuerdo a Evans (2002), que el que lo haga un hombre es visto con suspicacia, ya que implica que los motivos para el contacto físico no necesariamente son para dar confort o cuidado, sino más bien de naturaleza sexual.

Este último reto de Lupton, también se relaciona con lo que Heikes (1991) llama percepción de "identidad deteriorada". Los hombres accionan dos mecanismos, por un lado, hacen un esfuerzo por mantener su identidad masculina (Villeneuve, 1994) y por el otro, accionan prácticas para flexibilizar su masculinidad. Adicionalmente, los hombres generan prácticas para enfatizar y reafirmar su sentido de sí mismo como hombre, como asistir a sus colegas mujeres con trabajo pesado en el cuidado de los pacientes, intervenir en situaciones de pacientes violentos y evitar realizar actividades consideradas como femeninas (Evans y Frank, 2003).

Por otro lado, Williams (1992 y 1995a) ha documentado los privilegios que presentan los hombres por su género en las profesiones feminizadas. A este fenómeno se le ha descrito como glass escalator en el cual los hombres en profesiones feminizadas ascienden más rápido que las mujeres en profesiones dominadas por los hombres. En su estudio, Williams (1995a) observa que enfermeros tenían una media similar de salarios respecto de las mujeres, pero en el caso de los trabajadores sociales, ellos ganaban más que ellas. Evans (1997) también señala, que incluso en ocupaciones dominadas por mujeres, existen relaciones que denomina patriarcales. És- 
tas se reflejan en una alta valoración a todo lo que es masculino, lo que genera, que exista un desproporcionado número de hombres en posiciones administrativas y de élite. Williams (1995b) sostiene que en algunos casos se anima a los hombres a seguir carreras administrativas para evitar los estereotipos provenientes de las valoraciones de los pacientes. En este caso, los estereotipos negativos pueden presionar a los hombres para moverse "hacia arriba" en la profesión. Esto es consistente con el hecho de que los enfermeros hombres tienden a tener más grados universitarios que sus colegas mujeres (Greenberg y Levine, 1971; Okrainec, 1994).

De la revisión de estudios previos se observa que, estos se han enfocado principalmente a los enfermeros y que en ellos se han estudiado básicamente las desventajas asociadas a la percepción de su identidad masculina y las disparidades entre hombres y mujeres. En el presente artículo se incorporan más profesiones además de la enfermería, se analizan las desventajas que los hombres reportan, a través del grupo de hombres en sí mismo (sin considerar la comparación con las mujeres) y se busca presentar una perspectiva integral del rol de los hombres como cuidadores, tanto en su espacio laboral como en su participación en tareas de cuidado en el hogar. Nos interesa problematizar la feminización del cuidado, sin ignorar relaciones de poder y la violencia simbólica detrás de la división sexual del trabajo.

Este último punto lo hemos abordado a través de hombres que asumen un rol de cuidador en el espacio doméstico, distinto al de mero proveedor económico (Figueroa y Franzoni, 2011; Figueroa y Flores, 2012). Se trata de cuidadores de hijos, padres y parejas, a los cuales se les preguntan los motivos para convertirse en cuidador y sus percepciones sobre el rol de proveedor económico, cambio de valores y el significado que los hombres le dan a su masculinidad y su paternidad.

Este artículo es parte de un estudio multipaís (Brasil, Chile, India, México y Sudáfrica) que incorpora las experiencias de hombres cuidadores y en profesiones de cuidado (Barker et al., 2012). Algunas de las profesiones de cuidado asociadas a mujeres, de las que los hombres participan, son: profesores de preescolar, activistas, terapeutas, enfermeros, parteros, estudiante de medicina obstétrica, psicólogo, bailarín, vendedor, dueño de gimnasio, mehndi, ${ }^{3}$ director de escuela y profesor de música, entre otras. En la sección de hallazgos preliminares se recuperan algunos de los resultados de esta investigación multipaís a manera de punto de comparación,

\footnotetext{
${ }^{3}$ Es el oficio de aplicación de tatuajes de henna temporales, especialmente como parte de la preparación de la novia o del novio para una boda.
} 
pero en especial como propuesta de proceso reflexivo. Es decir, más allá de dimensiones cuantitativas, se propone explorar algunas prácticas de cuidado desarrolladas por hombres en oficios donde suelen ser minoría, no se trata de victimizarlos, sino de explorar acercamientos a prácticas que podrían ser transgresoras, pero a la vez formas veladas de ejercicio de la violencia simbólica (Bourdieu, 2000).

Desde los aprendizajes de género, se ha enfatizado el papel de los varones en labores extradomésticas remuneradas y en la contraparte, se asume que las mujeres son las encargadas de labores domésticas y del acompañamiento cotidiano de los integrantes del hogar en dicho espacio. Algunas teóricas de la categoría de 'cuidado' (Tronto, 1993) reconocen etapas en el proceso del cuidado, desde conseguir los recursos para que otros cuiden, hasta el hecho mismo de que una persona es cuidada y recibe atenciones por parte de otros, por lo que cada etapa engloba actividades convencionalmente asignadas a personas de ambos sexos. A pesar de ello, la literatura ha mostrado inequidades en la experiencia de las mujeres al centrarse en actividades no remuneradas económicamente, a la par que se asume que las labores de proveeduría económica - asociadas con los aprendizajes de género de muchos varones-, llevan en sí mismo la retribución, tanto por el ingreso como por el reconocimiento social. Sin embargo, menos se discuten las retribuciones emocionales de quien realiza lo doméstico y las demandas por ausencias constantes de quien provee económicamente. No son equivalentes, pero tampoco se han documentado los costos en el proceso de salud y enfermedad de quienes concentran su quehacer social en la proveeduría económica (hombres o mujeres), al indagar por lo que podría denominarse salud y mortalidad parental en un sentido amplio, a partir de sus respectivas asignaciones de género. En este caso nos interesa escuchar lo que van narrando los hombres en términos de gratificaciones, tensiones y ambivalencias en su entorno laboral, para luego dialogar con la literatura al retomar los relatos de los mismos sujetos dentro de sus respectivos entornos domésticos.

\section{Metodología}

El estudio comprendió siete entrevistas a varones que incluyeron tres enfermeros, dos profesores de educación preescolar, un profesor de primaria y un profesor de música de niños. El rango de edad de los entrevistados fue de 19 a 46 años con un promedio de escolaridad acumulada de 16 años. Al momento de la entrevista, tres de ellos estaban solteros, tres en unión y uno recién divorciado. Las entrevistas se condujeron entre agosto de 2009 
y noviembre de 2010 y los interlocutores fueron seleccionados como una muestra intencional en la que se incluyeran diferentes prácticas remuneradas de cuidado, pero en las que además los hombres fueran minoría. ${ }^{4} \mathrm{Se}$ buscaba una diversidad de casos, con el fin de poder reflexionar desde su heterogeneidad (Flyvbjerg, 2006). Las características de los entrevistados se presentan en la Tabla 1. La entrevista contuvo seis módulos: familia de origen, infancia y adolescencia; edad adulta y relación de pareja; familia actual; elementos de su vida social; ocupación actual y; políticas públicas. En este texto se enfatizan los hallazgos asociados con su ocupación, la forma en que llegaron a esta, su experiencia y sus tareas de cuidado, más allá de sus profesiones; es decir, sin restringirse al espacio laboral incursiona también en el hogar, con la intención de problematizar la forma en que se aprende, se ejerce y a la par se cuestiona y transgrede, la división sexual del trabajo. ${ }^{5}$

La investigación se trata de un estudio de caso (Flyvbjerg, 2006), ya que reúne la experiencia de siete hombres en profesiones de mayor participación femenina. Se generó un análisis inductivo a través de un proceso de tres etapas: i) se realizaron dos lecturas para codificar las entrevistas, ii) se utilizó un sistema de códigos que derivó de la bibliografía de hombres en profesiones feminizadas y cuidado y iii) se generó el reporte de los resultados.

Las entrevistas fueron hechas por una mujer y esto es relevante de comentar. La literatura ha encontrado que los relatos de los hombres sobre temas que se distancian de los estereotipos de género, son reconstruidos diferencialmente dependiendo si se comentan con un hombre o a una mujer (Figueroa, Jiménez y Tena, 2006). No se pretende afirmar que alguna sea más válida o verídica, pero sí que las representaciones sobre las valoraciones potenciales desde la escucha, permean los elementos que se destacan en los recuentos compartidos (Schwalbe y Wolkomir, 2001). Las transcripciones fueron analizadas con el apoyo del software Atlas.ti., lo que permitió sistematizar los resultados derivados de las entrevistas diseñadas y también poder captar hallazgos emergentes. El análisis utilizó un set de 443 códigos. Estos se agruparon en familias con el objetivo de poder identificar los componentes de los conceptos y los vínculos entre los mismos.

\footnotetext{
${ }^{4}$ Las entrevistas fueron realizadas y transcritas por Josefina Franzoni, a quien se le agradece este trabajo.

${ }^{5}$ Para un análisis más detallado de su contexto y del proyecto de investigación en general, pueden verse Figueroa y Franzoni, 2011; y Figueroa y Flores, 2012.
} 


\begin{tabular}{|c|c|c|c|c|c|c|c|}
\hline 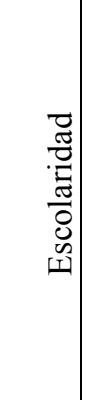 & 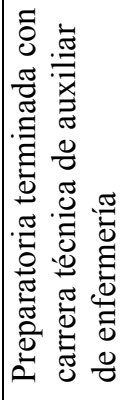 & 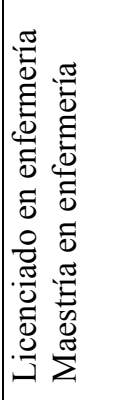 & 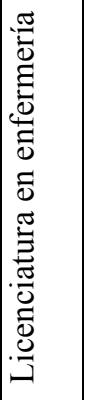 & 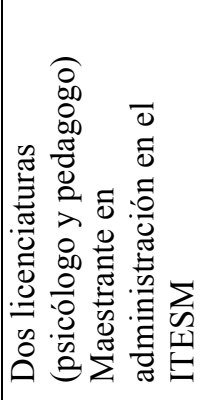 & 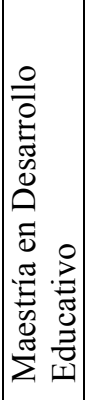 & 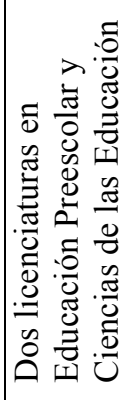 & 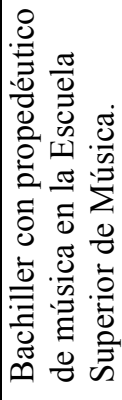 \\
\hline$:$ & z & 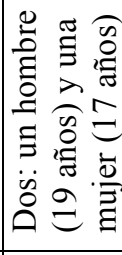 & 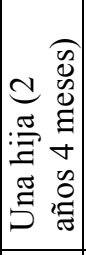 & 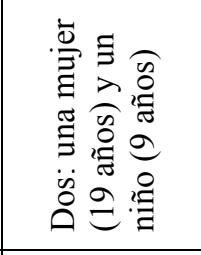 & $\stackrel{\circ}{z}$ & $\begin{array}{l}0 \\
0 \\
0 \\
0 \\
0 \\
0 \\
0 \\
0\end{array}$ & $\stackrel{\circ}{z}$ \\
\hline 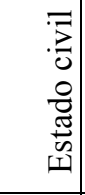 & 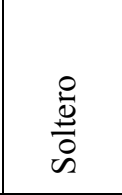 & 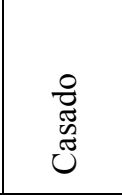 & 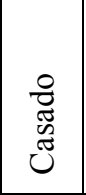 & $\begin{array}{l}0 \\
\frac{\pi}{0} \\
.0 \\
0 \\
0 \\
0 \\
0\end{array}$ & 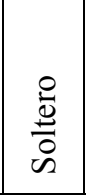 & 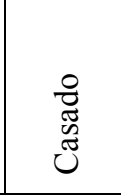 & $\begin{array}{l}0 \\
\stackrel{0}{0} \\
\frac{0}{0} \\
0\end{array}$ \\
\hline $\begin{array}{l}\vec{T} \\
\underset{I}{\tilde{I}} \\
\end{array}$ & 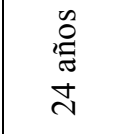 & 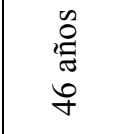 & 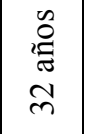 & $\begin{array}{l}\mathscr{0} \\
\stackrel{ే}{\Xi} \\
\stackrel{+}{ナ}\end{array}$ & 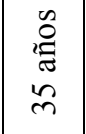 & $\begin{array}{l}\tilde{0} \\
\text { ఏే } \\
\text { యे }\end{array}$ & $\begin{array}{l}\mathscr{0} \\
\stackrel{\Xi}{\Xi} \\
\stackrel{\Xi}{=}\end{array}$ \\
\hline 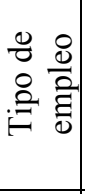 & 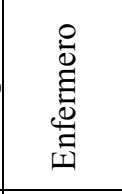 & 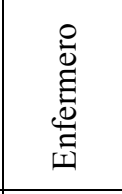 & 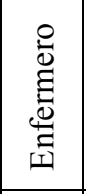 & 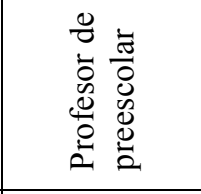 & 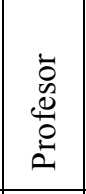 & 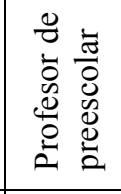 & 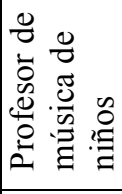 \\
\hline 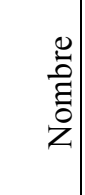 & $\begin{array}{l}\stackrel{0}{0} \\
\stackrel{0}{0} \\
\stackrel{0}{0}\end{array}$ & 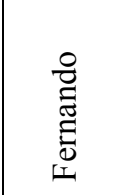 & 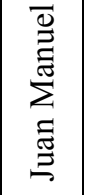 & 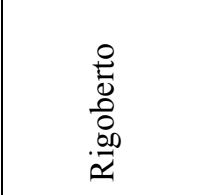 & $\stackrel{\mathscr{\Omega}}{\Xi}$ & בี & 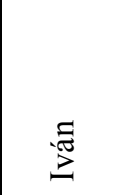 \\
\hline $\mathrm{Z}$ & - & $N$ & $m$ & $\nabla$ & $n$ & 6 & $r$ \\
\hline
\end{tabular}




\section{LOS VARONES EN LAS PROFESIONES FEMINIZADAS, LLEGADA, EXPECTATIVAS Y SALIDA}

\section{Llegada a la profesión feminizada}

Los hombres sugieren llegar a profesiones feminizadas por expectativas económicas, por experiencia laboral en la profesión, por barreras educativas y por gusto a la profesión. Las expectativas económicas derivan de haber escuchado de familiares o amigos que "se ganaba bien" en la profesión "(...) me habían hecho el comentario de que los médicos ganaban bien y la enfermería también", "y dice que ganan mucho dinero" (Jorge).

El haber tenido experiencia laboral de una persona cercana en la profesión feminizada sugiere ser un elemento que marcó la elección profesional futura. Tal fue el caso de uno de los entrevistados que tuvo que sustituir a su tía, quien era profesora de primaria. ${ }^{6}$ Después de esta experiencia, al entrevistado le gustó la profesión, motivo por el cual decidió dejar la carrera de ingeniería para entrar a la Escuela Normal Superior “(...) y no lo veía tampoco por esa cuestión económica o de prestigio, no, no lo hice por eso y no deje la otra por esa cuestión yo creo que era más lo que uno quiere y de verdad pues, le guste esa profesión (...)" (Luis).

Otra de las posibles causas por las que los varones llegan a las profesiones feminizadas es por barreras educativas. Esta situación se expresa cuando no se cuentan con las suficientes credenciales educativas o puntajes para acceder a su opción ideal y, por lo tanto, se tiene que escoger aquella opción que está disponible, dados los recursos educativos con los que cuentan. Por ejemplo, el caso de uno de los entrevistados que no terminó el bachillerato, pero estudió enfermería como carrera técnica:

(...) pues a lo mejor sí, pero me salí y me arrepentí, dije a lo mejor si hubiera terminado bachilleres, hubiera podido hacer el examen y entrar a la Facultad de la UNAM $^{7}$ o al Poli ${ }^{8}$ o a la UAM. ${ }^{9}(. .$.$) entonces dije bueno, vamos a in-$ tentar medicina, pero ya me dijeron, sabes que no se puede, dije bueno este pues ni modo no se pudo, entonces sea como sea, ya después entré a estudiar enfermería y ya trabajando me encantó, me apasionó (Fernando).

\footnotetext{
${ }^{6}$ La tía de este entrevistado estuvo incapacitada y dado que no había nadie que la sustituyera en la escuela, ella le pidió favor al entrevistado de hacerse cargo del grupo.

${ }^{7}$ Universidad Nacional Autónoma de México.

${ }^{8}$ Instituto Politécnico Nacional.

${ }^{9}$ Universidad Autónoma Metropolitana.
} 


\section{Expectativas de los varones y de sus familiares}

Si bien las expectativas económicas fueron señaladas por los entrevistados, pareciera haber una tensión en el planteamiento que depende de la trayectoria de los varones en la profesión. Es decir, sus expectativas económicas cambian cuando ejercen la profesión donde ahora se buscan un mejor nivel de vida y estabilidad laboral. Como ejemplo, el caso de uno de los entrevistados que reportaba mayores ingresos como vendedor que como maestro.

"Mi mamá me decía, es que me das más dinero cuando vendías ropa que ahora que me metí a trabajar de maestro, pero dije yo, es que eso no es para mí" (Rigoberto).

Sin embargo, en la medida que los varones se estabilizan en su trabajo, cambian su visión de la remuneración económica y ponderan otros elementos como lo son prestaciones laborales y la cercanía del lugar de trabajo con respecto al hogar "(...) es digamos a lo mejor no tener dinero, pero sí tener un mejor nivel de vida, ese es uno de mis anhelos, sobre todo. Porque mi mamá si sufrió bastante por ese motivo" (Fernando).

yo a través de estos años, no me he movido por el dinero, porque realmente lo que hemos ganado no es mucho pero afortunadamente los dos (su pareja y él) trabajamos; entonces hemos podido hacer muchas cosas juntos, los dos fuimos especialistas entonces con esos sueldos hemos podido hacer muchas cosas y no nos mueve el dinero a estas alturas (Fernando).

La familia ejerce presión en los varones que entran a las profesiones feminizadas. La entrada es vista por los familiares con suspicacia y preocupación respecto del futuro económico que el varón podría tener. Los familiares emiten valoraciones a través de la comparación entre los ingresos esperados en profesiones masculinizadas respecto de las profesiones feminizadas. No hacer caso de las advertencias de los familiares trajo a los varones problemas intrafamiliares. Por ejemplo, el caso de uno de los entrevistados, en donde el padre del varón quería que estudiara ingeniería:

(...) que primero que lo había defraudado que cómo era posible que hubiera dejado una carrera que me daba mayor prestigio, que me daba mayor cuestión económica, no recuerdo bien, sus palabras, pero sí me dijo que era una profesión de mujeres o de gente muy pobre económicamente (Luis).

\section{Movilidad en el empleo}

Los varones en las profesiones feminizadas, reportan deseos de ascenso en la estructura laboral, pero al mismo tiempo, este deseo puede entrar en ten- 
sión en la medida que se sientan satisfechos con el trabajo más operativo. Esto último está asociado con el cuidado directo de las personas. Por ejemplo, el caso de uno de los entrevistados que, a pesar de que tiene las credenciales educativas para optar por un puesto administrativo, el gusto por el cuidado a los pacientes, retrasa la posibilidad de ascenso:

(...) de que salí de la maestría que fue el año pasado, por estas fechas, precisamente yo siempre trabajé de operativo, nunca me interesó un puesto de jefe ni de supervisor. Aunque tuve la oportunidad de concursar y por los estudios que he tenido, porque antes de la licenciatura y de la maestría, hice infinidad de cursos y aparte hice una especialidad en neurología...; por tiempo, por experiencia y por logros académicos, hubiera podido concursar, hubiera ganado un puesto de jefe, pero no me interesaba, a mí lo que me interesaba es estar con contacto con la persona enferma (Fernando).

Este mismo deseo es reportado por otro de los entrevistados. En este caso, él había pasado varios años en puestos administrativos y reportaba el deseo de regresar al cuidado de los pacientes. Sin embargo, al mismo tiempo sus credenciales educativas lo posibilitaban para seguir dentro de los cargos administrativos.

Regresar un poco a mis orígenes, en el sentido de tener un trato más directo con el paciente, eso no se me dio y como que es lo que busco en estos momentos. A dónde me vaya a llevar mi preparación académica lo desconozco, porque igual se me da la oportunidad en un área administrativa (Juan Manuel).

En síntesis, más allá de limitarnos a constatar ciertos comportamientos esperados por la división sexual del trabajo asociada a diferentes referencias culturales, es de destacar algunos ejercicios de transgresión individual.

\section{VENTAJAS Y DESVENTAJAS DE LOS HOMBRES EN PROFESIONES FEMINIZADAS}

\section{Ventajas}

Las ventajas que reportan los hombres en sus profesiones se pueden distinguir mayormente en dos grupos: las personales y las relacionales. Las personales se refieren a todos aquellos elementos de desarrollo personal y profesional que asocian con el ejercicio de su profesión. Las ventajas relacionales se refieren sobre todo a la percepción y satisfacción de las relaciones interpersonales en el ámbito profesional. No obstante, pareciera ser que los hombres en profesiones feminizadas, asocian las ventajas de su 
profesión con motivos personales, como sentirse cómodos en el trabajo, el desarrollo en su carrera y la adrenalina que se vive en el entorno laboral, tan sólo por poner algunos ejemplos.

Una de las ventajas que reportan los hombres respecto de las mujeres dentro de su profesión es su fuerza física. Esta es usada para mover objetos pesados, pero también para facilitar el trabajo que requiere contacto físico. Este elemento es percibido por los varones como contrapuesto al trato que podría tener una profesional.

¿cómo le diré? Somos más toscos en cuestión de tener más actitud en manejar unidades críticas a los pacientes, porque no nos tocamos mucho el corazón, sino que hacemos las cosas como deben de ser, no es que " $i$ Ay le va doler!" o "al hacer algún movimiento la vayamos a lastimar". Como que los hombres somos más seguros a ese problema o ese servicio (Jorge).

Otra de las ventajas que es reportada por los varones,,$^{10}$ es mayor conocimiento de ciertos temas, sobre el conocimiento que pudieran tener las mujeres dentro de la profesión. Asimismo, se ve como una ventaja el carácter fuerte del hombre o varón para "curtir" a los estudiantes de las experiencias que vivirán en el siguiente grado escolar:

(...) en la mayoría de las escuelas he sido de los dos únicos varones y siempre nos dan los grupos mayores, para controlarlos, para ser más enérgicos, supuestamente también para prepararlos, o sea, es decir que nosotros tenemos mayor conocimiento en matemáticas, en español, en todas estas cuestiones y que como ellos ya van a enfrentarse a secundaria pues que de una vez ya vayan preparados y curtidos con nuestro carácter para enfrentar esas cosas (Luis).

Otro elemento que los varones reportan como una ventaja de su profesión es la adrenalina. En este caso se trata del ámbito de la enfermería donde ciertas especialidades, como lo es el área de urgencias, requieren de atención rápida y oportuna. Esta sensación incluso se puede trasladar a espacios no profesionales.

Porque teníamos pacientes que se ponían mal en un ratito entonces teníamos que andar así, entonces llegaba el paciente en la ambulancia y atenderlo rápido, o sea, como luego se ve en las series de televisión, así andábamos luego,

${ }^{10}$ A partir de este momento optamos por hablar de varones con el propósito de alertar sobre el uso acrítico de hombres como sinónimo de humanidad y a la par como referencia a los sujetos del sexo masculino, ya que con ello se tiende a ocultar discrecionalmente a la población femenina. No obstante, en esta sección del texto intercalamos los términos varones y hombres para facilitar la lectura del mismo. 
entonces es esa adrenalina. Yo les comento a otras personas, amigos, que por ejemplo algunos son adictos a la adrenalina (Fernando).

La misma actividad de cuidado es vista con satisfacción, lo cual contribuye a cuestionar algunos estereotipos sobre la incapacidad o desinterés de los hombres para el cuidado. En particular, los enfermeros reportan gusto por ayudar a los pacientes. La gratitud de los pacientes es vista como una evidencia del esfuerzo de su trabajo. La recuperación de los enfermos es visto como un bien valioso y también es en ellos que pueden reflejar el valor de la vida.

(...) con los pacientes, yo estoy muy agradecido porque me han enseñado mucho, me han enseñado que un día vivido, es un día que uno debe disfrutar, un día más he tenido pacientes con cáncer, con VIH y uno los ve y dice no puede ser que esta persona este contenta si sabe que en algún tiempo más se va a morir, pero no, o sea la sorpresa es esa que ellos viven tranquilos (Fernando).

Entre las ventajas relacionales que reportan los varones en sus profesiones se encuentran: el desarrollo de las personas a las que cuidan, el trato con mujeres y el poder integrar diferentes puntos de vista para la toma de decisiones. El aprendizaje de los menores, es visto como un bien valioso por los hombres enfocados a la educación. El desarrollo de los menores durante el proceso de aprendizaje, es reportado también con detenimiento y ven a los menores como sujetos susceptibles a ser moldeados "de ver ese niño que no sabía caminar y tú lo ves que ya camina, de ver ese niño que no sabía hablar y ver que ya te habla, ver ese niño que no quería comer, no quería comer verduras y ahora las come y pide más" (Rigoberto).

Por otro lado, dentro de los enfermeros se reportó el gusto por la convivencia con mujeres, con aseveraciones como ambientes de trabajo tranquilos y cómodos por la presencia de mujeres. La convivencia con las mujeres es vista como un bien, en comparación con otras profesiones con menor prevalencia femenina, lo cual no pretende ignorar los costos de la profesionalización de la carrera y los posibles beneficios que ello les genera a los hombres, pero sí evidenciar los relatos de ellos al dar cuenta de su incursión en estos espacios.

(...) para mí significa mucho, como le digo, una mujer y me encantan las mujeres, no solo físicamente, sino porque puedo platicar también con ellas de muchos temas; a pesar de todo, muchas veces no las he logrado entender a la perfección, pero yo creo que si me complemento muy bien, para trabajar con ellas. Yo digo si soy afortunado, la verdad (Fernando). 
Una tercera ventaja que fue reportada por los varones, es la posibilidad de poder integrar diferentes puntos de vista en la toma de decisiones. Esto puede ser interpretado de dos formas, la primera que sea un estilo de gobierno en donde los hombres en puestos de jerarquía busquen la opinión de sus subalternos. La segunda interpretación que podríamos tener es, que la composición del grupo de subalternos, que en este caso son en su mayoría mujeres, sea la causa por la que ellos busquen estas voces y en donde el varón al mando podría minimizar sus ventajas de género con la integración de participación femenina en la toma de decisiones. Esto puede tener lecturas contrastantes desde la violencia simbólica, transgrediéndola o bien reproduciéndola.

(...) conocer, aprender de mis compañeros, de las relaciones que establecemos, que me permite ver como diferentes perspectivas y no solo quedarme con mi perspectiva; cuando estaba como profesor frente al grupo yo solo veía mi percepción y no me interesaba la percepción que tenía de mi los chavitos, de los padres de familia de mis demás compañeros y estar ahorita como director es esa satisfacción de poder ver de humanizarme más, como los intereses que tienen todos en conjunto no solo centrarme en mi visión (Luis).

\section{Desventajas}

Los varones reportaron desventajas dentro de su profesión en múltiples niveles. De estos se pueden identificar cinco áreas: autolimitación, relaciones en el espacio de trabajo (compañeros), relaciones con sus beneficiarios (menores y pacientes), relaciones con beneficiarios indirectos (padres de familia y familiares de enfermos) y entorno institucional.

Se observa en un primer nivel, la autolimitación de los varones para ejercer plenamente su profesión respecto de las mujeres que también son parte de esta. Las causas de estas limitaciones pareciera que vienen dadas por el desagrado a determinadas actividades, por el pudor en el trato con menores (mujeres), y por un conocimiento previo del entorno de trabajo del que son parte. Del primer ejemplo, se tiene que uno de los profesores prefería no tener grupos de los primeros grados de primaria, por el desagrado que le implicaba el cuidado de la higiene de los menores: "yo no vería como hombre, limpiándole los moquitos a los niños, o acompañándolos al baño" (Luis). Del segundo elemento, se tiene que uno de los profesores reportaba mayor facilidad para hablar con los niños que con las niñas: Sí, sí, pero yo no sabía (risa) o sea digo uno también es como pudoroso al principio de la situación, digo con las mujeres, con los chavitos no me costaba ningún trabajo, pero con las niñas sí (Luis). 
Del tercer elemento, uno de los enfermeros reportó preferencia a la asignación de pacientes varones. Con ellos es más fácil atender sus necesidades, a diferencia de las pacientes mujeres con las que tendría que pedir ayuda a sus familiares: “(...) he tratado que me toquen hombres porque para apoyarlos y ya; sí me toca una mujer trato de que me apoyen sus familiares para que no haya tanto esa incomodidad" (Jorge).

El segundo nivel donde los varones reportan incomodidad es, en la relación con sus compañeros de trabajo, posiblemente por competencia entre pares. Estos problemas vienen dados por el incumplimiento de reglas informales y por percepciones de roles de género diferentes en el trabajo. El ejemplo que reportó uno de los entrevistados, es que su renuencia a bañar diariamente a los pacientes, le trajo conflictos con sus compañeras de trabajo:

(...) nos han enseñado siempre a respetar a la persona, entonces el hecho de que en este instituto el baño casi sea sagrado, crea muchos conflictos a veces, porque si a mí se me ocurría decir la señora o el señor, no se quiso bañar, casi me pegaban mis compañeras. Pero por ejemplo, yo lo veía de esta manera, si la señora o el señor no se querían bañar ese día ¿por qué lo iba yo a obligar a bañarse? Era su decisión que no querían, que tenían frío o no sé y a veces respetaba esas decisiones. Yo tuve muchos problemas al principio, precisamente por eso (Fernando).

A pesar de que el trabajar con mujeres fue reportado como una ventaja de la profesión, en particular el lidiar con el temperamento de las mujeres, fue reportado como un elemento problemático en las relaciones de trabajo: “(...) si porque es difícil el temperamento, el carácter de las mujeres es a veces este, bueno yo no digo que inestable, pero a veces si es muy cambiante, entonces este lidiar con diferentes caracteres es difícil" (Fernando).

Sin embargo, en contraposición con el gusto por trabajar con mujeres, el compartir el espacio de trabajo con otros varones pareciera ser menos agradable. La falta de aceptación y la imposibilidad de establecer relaciones de trabajo fueron dos de los elementos enfatizados por los entrevistados:

(...) llegué a una Escuela donde sólo había dos varones, pero todos grandes y la mayoría mujeres, ¿por qué hago referencia a esos dos varones?, porque de alguna forma como llego chavo, la mayoría tendría de 46 a 47 años para arriba, me aceptaron muy bien, aunque los varones no (Luis).

(...) las mujeres (enfermeras) son así, como que tratan de congraciarse con ellos (médicos). Y eso también a ellos les disgusta, esperarían de los enfermeros el mismo trato, digamos que los hombres ¿son un poco más dignos? 
Los enfermeros son más dignos en términos de que, hacen su trabajo y no les importa estarse congraciando con el médico (Jorge).

Por otro lado, los varones en profesiones feminizadas reportaron tensiones en los roles de género que les son asignados. Por una parte, emerge la percepción de que como los hombres son autoritarios y rígidos al ejercer la disciplina, pueden atender casos más críticos; por otra, se llega a interpretar que la convivencia frecuente con mujeres genera afeminamiento en sus comportamientos y con ello se les califica como homosexuales. Por poner un ejemplo, de la demanda del rol autoritario, se tiene que, a uno de los profesores le son enviados los menores para que sean castigados, a pesar de que este no era el estilo del profesor. Es decir, también viven las consecuencias de imágenes rígidas del ser hombre:

Pasó cuando subí de Director me paso con los chavos, sobre todo porque en esa escuela eran muchos, había varios maestros varones, pero eran ya adultos, entonces los trataban con mucho autoritarismo. Luego llega una persona joven como Director, que además los apapacha; es decir, hay algún problema y siempre me los mandaba para que los reprimiera y toda esta cuestión (...) (Luis).

La otra tensión fue reportada por uno de los enfermeros, quien advierte una especie de "conversión" de los varones provocada por la convivencia con las mujeres, que se interpreta como que los hace convertirse en homosexuales, o al menos contar con características afeminadas: “(...) no se comportan como hombres o como varones y dan comentarios como que más afeminados. (...) Hay hombres que por tanta convivencia con mujeres se llegan a deteriorar, son más amanerados y llegan a ser homosexuales" (Jorge).

La demanda del medio, por relacionarse con mujeres es vista como un ritual, en donde el varón que llega al espacio laboral feminizado, tiene que "colonizar" relacionándose afectivamente con las profesoras, o bien con las madres de familia, por poner el caso de la escuela. Es decir, reproduciendo una imagen del sujeto sexual potente y activo, al margen de cómo lo viven sujetos en específico:

(...) de todo, y sobre todo porque me ven joven entonces si es como pus (sic) ya llego el gallo que tiene que pisarnos no y además es como un ritual en la escuela, no sé si en otras profesiones, pero en la escuela es como un ritual muy marcado, que por lo menos tenemos que andar con las madres de familia, a mí no me gustan estas cuestiones y sobre todo no me gusta tener problemas. Entonces al evitar los problemas pues después hablan de mi hasta lo peor; en la primaria nosotros somos acosados por las profesoras (Luis). 
Los varones también reportaron barreras de cristal en el espacio de trabajo. Ellos reciben beneficios por parte de las mujeres y además existen reglas informales de distribución del trabajo por sexo. El beneficio de los hombres, respecto de las mujeres, es reportado con consternación por parte de uno de los entrevistados, lo cual pareciera ponerlo en una posición de cuestionamiento de los mismos privilegios asociados a su ser hombre:

Entonces no quería ser ni más que ellas ni menos, quería ser igual digamos en el sentido del trabajo, inclusive había un estudiante que llegaba y le decía a la compañera lávame las palanganas para lavar a mi paciente, o sea, que por el hecho de ser hombre, creía que merecía que los demás hicieran las cosas por él y dije no, estás mal, para mi sorpresa había compañeras que sí lo hacían (Fernando).

Por otro lado, la distribución de los grupos por el sexo de la persona docente, fue reportada como una limitante, tanto para hombres como para las mujeres. Las profesoras mujeres son asignadas usualmente a los grupos de primer y segundo grado de primaria en los cuales, los menores aún tienen que aprender hábitos e higiene. Al contrario, los profesores varones son asignados a grupos de quinto y sexto grado de primaria, con la búsqueda de la formación del carácter y educarlos para los siguientes niveles educativos, lo que nuevamente constata la reproducción de estereotipos de género, incluso en términos de legitimar estigmas y a la par privilegios de relaciones jerárquicas:

(...) están tan acostumbradas a que sean mujeres que no perciben tanto que llegue otro hombre y por otro lado dentro de la escuela tienen las funciones como en el hogar, muy diferenciadas. Por supuesto que se juega el género en esta cuestión, como que cada uno lo asume (Luis).

En el tercer nivel, los varones - en particular los enfermeros-, reportaron incomodidad por la forma agresiva en que los pacientes se dirigen hacia ellos, pero al mismo tiempo reportaron sensibilidad ante la muerte de sus pacientes. Es interesante que, en el siguiente fragmento de la entrevista de Fernando, cuando este indica la afectación por la muerte de los pacientes, utilice el llanto de sus compañeras como una forma de denotar su propia sensibilidad ante una situación así.

(...) nos dolía cuando ellos estaban sufriendo y nos alegrábamos que iban evolucionando bien, un paciente que sale bien para nosotros es un triunfo, porque eso es para lo que nosotros estudiamos, eso es a lo que nos dedicamos, ese es nuestro sostén, nuestra vida. Si un paciente se muere, créalo que en la terapia 
ha habido compañeras que han llorado por pacientes porque se mueren porque nos llega a lastimar no solamente en la terapia sino en cualquier servicio" (Fernando).

En este mismo tercer nivel, los varones en profesiones feminizadas reportaron problemas y malestares por tratar con mujeres, incluidas estudiantes y pacientes. Por parte de las estudiantes, existe el temor de que ellas los acusen de abuso y, por consiguiente, que los padres generen sospechas al respecto. Esta presión es vista con molestia por parte de los profesores, que tienen que tener cautela en la relación cotidiana establecida con las menores:

(...) prefería pedirle a alguna profesora que los llevara, sobre todo con las niñas, estaba temeroso como varón, yo creo que si bien es cierto que hay violaciones y toda la cuestión pero tocar a una niña se puede interpretar más y yo tenía miedo a esa cuestión, porque en esa escuela que estuve, que me toco ese grupo de segundo que eran profesores universitarios se habían dado problemas muy fuertes, donde los profesores que estaban en primero o en segundo tocaban a las niñas, pero no las tocaban maliciosamente (...) (Luis).

En particular, los enfermeros, reportaron el rechazo de las pacientes mujeres a ser atendidas por un varón. Este rechazo es más común en pacientes de la tercera edad, que piden ser atendidas por una mujer, en parte por los referentes simbólicos asociados a la construcción sexual de las diferencias corporales. Los varones consideran esta renuencia, como una barrera para ejercer su profesión a cabalidad.

A las señoras de la tercera edad que sean mujeres siempre, "que me bañe una mujer, que me atienda una mujer" porque hombre no quiere prácticamente; a veces hay esa barrera y yo trato de ganármela porque el día de mañana que toque pues tengo que tratar con ella (Jorge).

En el caso contrario, los enfermeros reportan que los pacientes varones son más accesibles a ser atendidos indistintamente, ya sea por una enfermera o por un enfermero. "No para nada, los hombres son más accesibles y no importa la edad son más accesibles y son más tímidos en cuestión de compañeros, pero son más mente abierta; a ellos no les interesa que los vea una enfermera" (Jorge).

En ambos casos, con las estudiantes y con las pacientes, los hombres tienen que cuidar el contacto físico. Pareciera ser que los hombres se refieren a dos tipos de contacto: el contacto con una intención sexual y el contacto por cariño cotidiano. No obstante, que la diferencia no es clara; pareciera que los varones, quisieran poder establecer contacto físico del 
segundo tipo con mayor tranquilidad, sin ser acusados de abusadores en término sexuales.

En un cuarto nivel, los varones se ven presionados por los padres de familia y por los familiares de los pacientes. Las madres y padres de familia demandan un trato más "cariñoso" hacia sus hijos pequeños (primeros grados de primaria), pero al mismo tiempo, limitan el abordaje de ciertos temas por parte de los profesores hacia las estudiantes.

(...) aunque yo no les hablaba fuerte, pero sintieron ese cambio, o sea son niños de segundo que de alguna forma vienen de estar con puras profesoras entonces si un cambio con profesor varón, si es difícil para ellos y las mamás mismas en algún momento me lo externaron, que tratara de ser un poco más cariñoso con ellos, entonces tuve esa experiencia, fue una experiencia bonita, pero yo creo que nunca la volvería a repetir, por esa misma situación (Luis).

La limitación que ponen los padres, se relaciona con el abordaje de temas de sexualidad. Ante esta limitante, los profesores tienen que hacer una división del trabajo por género, donde los profesores hablan con los estudiantes y las profesoras con las estudiantes para abordar los contenidos de sexualidad.

(...) creo que para nosotros como varones trabajar con niñas es más complicado porque las mamás y los papás nos exigen que llevemos a alguien, si van a ser mujeres es que es bien chistoso ahorita (risa) Si les vamos a dar educación sexual a las niñas, tenemos que llevar a una mujer que hable con ellas (...) (Luis).

Finalmente, en un quinto nivel, los varones refieren presiones del entorno para ejercer su profesión. Uno de los enfermeros reportó, que existen hospitales que no aceptan varones: "que no, que sólo puras mujeres" (Jorge). Por otro lado, uno de los profesores reportó que el entorno de violencia, no es proclive para el aprendizaje de los menores y, por lo tanto, esto tiene un impacto negativo para ejercer la profesión:

También el entorno de la escuela es un factor muy complicado, sobre todo en la escuela esta de Santo Domingo; el entorno era muy complicado o sea, este sabíamos que muchos de los niños no se iban a su casa a veces uno tenía que cuando salías los veías y decirles oigan váyanse a su casa, si quieren los llevo, los acompaño y cualquier cuestión para que se fueran a su casa y no se quedaran como con banditas que estaban en el entorno. Entonces yo creo que el problema no son los niños, somos nosotros como adultos y la interferencia que 
hacemos y también el contexto por supuesto influye alrededor de la escuela (Luis).

\section{Arreglos de Cuidado en CASA de los hombres EN PROFESIONES FEMINIZADAS}

Los varones en profesiones feminizadas, cuidan en el ámbito familiar de hijos, madres y padres. El varón en una profesión feminizada pareciera ser, que es más proclive a que le sea asignado el rol de cuidador en el espacio familiar, a pesar de los imaginarios sociales en sentido opuesto (Barker et al., 2012). Por otro lado, dentro de su profesión los varones reportaron haber ido más allá de su práctica, y haber activado respuestas para comunicarse con los menores y enfermos, de tal forma que, estos se sintieran cómodos y escuchados. Ante contextos complejos, los varones reportaron generar formas de adaptación ante las circunstancias, para lograr conectar con las personas que debían ser cuidadas.

\section{Cuidado de hijos, madres y padres}

En el cuidado de hijos pequeños, menores de siete años; se observa que existen cambios de horario en la jornada laboral, para poder asumir el rol de cuidador de sus hijos, lo cual diversifica la figura del progenitor masculino en labores de cuidado (Figueroa, 2018). Uno de los enfermeros cambió su turno a nocturno para cuidar de sus hijos por el día. Esta decisión, es vista como una diferencia, respecto a lo que el enfermero consideraba que es el rol tradicional que asumen los papás. Como correspondencia de esta acción, el enfermero expresa satisfacción por haber visto a sus hijos crecer:

(...) de hecho cuando mis hijos estaban chiquitos, Tania tenía siete años, yo la crié prácticamente, sí. Entonces yo a ella la ví dar sus primeros pasos, yo a ella este, oí sus primeras palabras, este, yo la bañaba, yo la cambiaba; entonces yo creo que son momentos que muchas veces los padres hombres se pierden, pero gracias a que yo estuve trabajando en la noche, me tuve que hacer cargo de mis hijos en el día, yo creo que gracias a eso pude disfrutar el crecimiento de mis hijos (Fernando).

En el caso de otro enfermero, se reporta el deseo de querer cambiar su horario laboral, para estar más tiempo con su hija de dos años y con su esposa.

En ocasiones llego a ver a mi hija, en ocasiones no; la gran mayoría no, ya la veo dormidita. Casi se hace cargo de ella por completo mi esposa, en darle de 
comer, estar con ella; es eso por lo que incluso quiero buscar otro trabajo que tenga menos horas y que permita convivir más con mi familia (Juan Manuel).

El cuidado de personas ajenas al hogar, pareciera ser un elemento detonador de problemas de pareja. Esto se expresa a través del celo de cuidar de alguien más y no a los propios miembros de la familia. Por ejemplo, el caso de uno de los profesores de preescolar, que reportó celos de su pareja porque él cuidaba de los niños de la escuela y no "cuidaba" de su propio hijo:

Cuando llega el otro niño ya estábamos en el colegio porque nosotros empezamos a trabajar en mayo y ella se alivia en septiembre ¿aja? ¿Entonces qué pasa? Pues digo bueno mis clientes son los niños yo tengo que tratar de cuidar a los niños entonces era los celos ¿Por qué le hace caricias a ese niño y no se las haces primero a tu hijo?, ¿Por qué no esto y no a tu hijo?, ¿Por qué? (...) (Rigoberto).

Los hombres en profesiones feminizadas, parecieran asumir un papel de guía de sus hijos, más allá de sólo asumir el rol de proveedor económico. Con este papel de guía, se pretende que sus hijos alcancen su desarrollo personal, con poco énfasis en los puntajes educativos. Por ejemplo, el caso de uno de los enfermeros que es padre de dos: un hijo y una hija.

Entonces para mí ser padre, más bien, no es el llevarles el bocado a la boca, el darles el dinero para que se compren lo que quieran es guiarlos y encausarlos para que sean personas de bien y que a su vez ellos puedan vivir una vida plena y que sean felices; más que un título o que un logro académico, con el hecho de que sean felices es más que suficiente. Y si lo logran con un título, pues qué mejor, pero si lo logran de otra manera, pero son felices, yo creo que es lo que todo padre quiere (Fernando).

A pesar de lo anterior, este vínculo con los hijos puede estar mediado por la relación con la madre. Por ejemplo, uno de los varones que estaba divorciado, reportó que su ex pareja manipulaba a sus hijos. Ante esto, el varón reportaba el anhelo de que sus hijos pudieran ser autónomos y pudieran tomar sus propias decisiones:

¿Qué espero realmente de cuando mis hijos sean grandes? Ya la considero a mi hija por supuesto un adulto en realidad; yo quiero llevar con ella una relación sobre todo donde no se deje manipular por nadie, independientemente de que sea su mamá, o de que sea su papá o sea su pareja. Sea quien sea, no se deje manipular es lo único y bueno con respecto a mi hijo, pues realmente que nos sigamos platicando todo, como llevamos hasta ahorita (Rigoberto). 
En este mismo caso, la separación de su expareja fue retrasada para poder mantener una relación cercana con sus hijos. Al final él decide separarse a pesar de que sabía que la relación con los hijos se podría deteriorar: "(...) se supone yo iba a ver a mis hijos los fines de semana, pero cuando ella quería los veía, cuando ella no quería no los veía (...) (Rigoberto).

Algo análogo fue lo encontrado por Jiménez (2003), al documentar conflictos en las relaciones de pareja; ella encontró varones que le comentaban que no se divorciaban mientras tuvieran hijos menores de edad, pues temían que se les impidiera seguir viendo a sus hijos. Por su parte, Perujo (2015) documenta la búsqueda que construyen hombres divorciados cuando se les impide ver a sus hijos, a partir de que la patria potestad no se les otorga.

Los varones en profesiones de cuidado, también reportaron cuidar de sus padres y madres. El cuidado involucraba sostén económico y trabajo físico, lo cual genera una dualidad interesante y compleja, ya que mientras que diferentes demandas feministas han logrado que el trabajo físico y en general el doméstico haya sido reconocido poco a poco como trabajo productivo, no es tan claro que el trabajo de proveeduría económica sea reconocido como cuidado, como lo sugiere la filósofa feminista Joan Tronto (1993). A pesar de ello, en algunos hombres entrevistados la presencia de enfermedad en los padres de los varones, parece ser, que fue el evento que detonó que ellos asumieran el rol de cuidador. "Fue como nuevamente fijarnos mucho en ella, que estuviera bien, que no hiciera nada, este entonces ahí cero que fue doloroso (...)" (Luis).

Dentro de las entrevistas, se reportó el uso de las redes profesionales y de los recursos laborales para brindar cuidado a sus familiares. Como ejemplo, se encuentra el caso de uno de los enfermeros, quien reportó el uso de sus vínculos laborales para que su madre recibiera atención médica, que incluyó medicinas y operación: "Y ya me fui al hospital al que yo trabajaba; ahí conseguí el medicamento que pedían y cosas, lo llevé al hospital. Y a mi mamá la operaron de contrabando (...)" (Jorge).

El hecho de que los varones hayan cuidado de alguno de sus padres, les asigna un rol de cuidador dentro de la familia. Se reportan presiones de los hermanos para que el varón cuidador se haga cargo del padre o madre que aún está con vida. Por ejemplo, el caso de uno de los varones quien reporta que, al morir su madre de la que él principalmente se hacía cargo, los hermanos querían que ahora se hiciera cargo de su padre, porque él era el único soltero de los hermanos y "no tenía responsabilidades". Él decide no tomar el papel de cuidador, porque ve a su padre autosuficiente, al contrario de la madre que estaba enferma: 
(...) llega un momento en el que digo no, no estoy haciendo mal, él ya es un hombre que tiene que valerse por sí mismo, ya es adulto y ahí es donde decido como, alejarme de él. Digo sí lo veo, es decir, estoy pendiente de él porque, él también está, tiene eh, tiene diabetes, curiosamente esta cuestión de mis hermanos se difuminan, porque nuevamente me echan a mí como la responsabilidad de cuidar a mi papá (Luis).

El no asumir el rol de cuidador de los padres, puede traer a los varones distanciamiento de su familia, que se expresa en dejar de hablar con sus hermanos y cambio de domicilio para evitar que le sean asignadas las tareas de cuidado. Sin embargo, el cuidado de los padres no necesariamente es visto como una carga o una responsabilidad asignada por los hermanos; también es visto como una actividad gratificante. Por ejemplo, uno de los varones reporta satisfacción al cuidar de su padre: “(...) además soy el único hermano que vivo con él, entonces yo como les digo este pues muéranse de envidia porque yo soy el que lo está disfrutando más" (Raúl).

\section{Cuidado dentro de sus profesiones (sensibilidad en el cuidado)}

Como practicantes de su profesión, los hombres brindan cuidado a enfermos (en el caso de los enfermeros) y niños y niñas (en el caso de los maestros). Se observa que este cuidado va más allá de las actividades profesionales establecidas; es decir, son acciones que pareciera que derivan más de asumir una conciencia como cuidador. Esta conciencia se expresa en dos vertientes, por un lado, buscan la estabilidad emocional de la persona a la que cuidan, pero también buscan comprender la complejidad de la situación por la que pasan los enfermos y los niños y dar una respuesta asertiva. En este sentido es interesante reflexionar con Connell (2015), quien señala que los hombres tienen alta probabilidad de perder un juicio por la custodia de sus hijos, dada la gran experiencia acumulada de las mujeres como cuidadoras. Si bien no especifica que dicha experiencia se ha generado en muchos casos por una imposición del papel de cuidadoras, valdría la pena reflexionar qué sentido les generaría a los hombres construir una experiencia como cuidadores, como para contrarrestar simbólicamente dicha "desventaja".

La comunicación entre los padres y los niños es un área en la que los profesores reportaron preocupación. Ante esta situación, los profesores activaban mecanismos para promover que los padres platicaran con sus hijos, pero también reportaron el establecimiento de canales de comunicación con los menores, a los cuales sus padres no les hacían caso. Como ejemplo 
de la primera idea, se tiene que uno de los profesores, reportaba platicar constantemente con los padres para mejorar las relaciones intrafamiliares:

(...) si platicas con padres... llevo esa parte de orientación como terapista, lo tengo que manejar ¿Qué les recomiendo mucho? Primero es conocerlos porque tengo muchos papás, les digo bueno y ¿cuál es su platillo preferido? o ¿el postre o la sopa preferida? Todos los días los ven comer (...) y no saben (...) (Rigoberto).

Los profesores activan canales de comunicación con los menores a través de dedicar minutos específicos de "plática libre" o espacios de debate grupal. Por ejemplo, uno de los profesores de música, quien reportaba preocupación por una de sus alumnas. Ella tenía diez años de edad y, de acuerdo al reporte del profesor, tenía necesidad de ser escuchada:

últimamente he platicado con ella y a ver ya Malenita, o sea, vamos hacer algo, como sé que no vas a dejar de platicar lo sé perfectamente tienes diez minutos al principio de la clase para platicarme lo que quieras, ya por lo menos entra la dinámica de mucho más trabajo... por ejemplo esta chica su papá tiene cuatro años sin trabajo, es un irresponsable y se deslinda de su hija (Iván).

$\mathrm{Al}$ igual que los profesores, los enfermeros reportaron la búsqueda del confort de sus pacientes. Se reportó especial énfasis en "respetar las decisiones de los pacientes", que se expresaba en momentos a solas y evitar baños diarios. Uno de los enfermeros reportó que, el respetar las decisiones de los pacientes, a veces traía problemas con sus compañeras de trabajo, toda vez que contravenían las reglas informales de la profesión:

con ellas tuve muchos problemas, porque aquí el baño es sagrado, si uno no baña a su paciente uh!, es como si no hubiera venido a trabajar. Pero yo lo he visto de otra manera, respetando siempre a la persona, porque a veces yo llegaba y los veía todos entumidos y ni modo de que lo iba yo a bañar en la mañanita, a veces me esperaba hasta medio día o simple y sencillamente, no me quiero bañar. Entonces tuve muchos problemas por respetar las decisiones de los pacientes (Fernando).

Las respuestas que brindan los varones en profesiones feminizadas para brindar cuidado pueden ser más complejas que la comunicación y el confort de niños y enfermos. Estas pueden implicar la comprensión de diversos elementos del entorno de los niños y enfermos y, la creación de una respuesta asertiva ante esta complejidad. Como ejemplo de esto, se tiene que uno de los profesores observó una situación de acoso escolar hacia uno de sus alumnos. Ante esta situación, el profesor tuvo que generar una 
respuesta para hablar de homosexualidad, con su grupo de sexto grado de primaria:

Yo no lo veía como algo que fuera como un problema grande, no lo hacía como un escándalo y un ultraje a la situación y escondan al niño y golpeen al niño, y toda esta cuestión, sino trataba de sensibilizar a todos y a todas de esa cuestión, o sea, de es natural déjenlo si él tiene esos gustos, es como ustedes que tienen gustos por las niñas o ustedes por los niños, digo es tan normal y natural (Luis).

Los varones en profesiones feminizadas reportaron haberse encontrado dentro de situaciones en las que los enfermos y los menores, no estaban siendo cuidados por sus familiares. Es decir, la red de cuidado se presentaba fragmentada. Esto, en el caso de los profesores, se expresaba a través de clara negligencia de los padres en el cuidado de sus hijos. Ante este contexto, uno de los profesores, activó una actitud más agresiva y autoritaria para poderse comunicar con sus alumnos y poder realizar las actividades de su profesión. De alguna manera, construyen estrategias de resistencia y transgresión a lo que socialmente está normado (Petchesky y Judd, 1998), al margen de cómo se nombran.

(...) aquí nadie te va a demandar porque los papás ni se preocupan con sus hijos, lo vas a ver cuando tengas la junta (palabras de la directora). Entonces yo llego al salón y les digo haber cabrones siéntense porque vamos a empezar a trabajar, los niños voltearon, porque fue para mí muy brutal porque los chavitos se voltean, las chavitas se voltean y se sientan, yo no sé cómo llamarle a eso, pero tenía que decirles cabrones, güeyes, o sea, no palabras tan altas, pero solo así pudieron hacerme caso y ver que también tenía autoridad porque además sus papás así les hablaban (Luis).

Finalmente, los mecanismos que los varones en profesiones feminizadas accionan para conectar con los menores y enfermos les traen retribuciones afectivas. Por ejemplo, uno de los profesores reportó que él disfrutaba de la compañía de los niños, más de lo que lo pudieran disfrutar sus propios padres. Esto se traducía en cariño de los niños hacia su persona:

(...) tienes que plantear un poquito más la figura de autoridad que debes de tener tú y con eso la verdad el niño entiende más, porque el día en que el niño llegue y que te de un abrazo, sin que le digan saluda, da abrazos, con nosotros llegan y te dan el abrazo y te dan el beso de una forma espontánea (Rigoberto). 


\section{REFLEXIONES SOBRE LOS HALLAZGOS}

En lo que respecta al primer eje de análisis, relacionado con la entrada de los varones a las profesiones feminizadas, el estigma de los padres hacia la profesión feminizada ya había sido señalado por Taylor (2012). Ella señala que los padres de los varones hacen exhortos a que ellos cambien de elección profesional y ante la renuencia de los varones, pueden presentarse problemas intrafamiliares.

Sobre el segundo eje de análisis, a propósito de las ventajas y desventajas de los varones en profesiones feminizadas, se encontraron similitudes con trabajos precedentes. Evans y Frank (2003) y Taylor (2012) señalan que los varones enfatizan sus aspectos masculinos tales como: fuerza física, conocimiento y carácter. Estos actos pareciera ser, que son llevados a cabo por los hombres para enfatizar y reafirmar su sentido de sí mismo como tales. Estos elementos fueron señalados por los varones en el presente estudio, con aseveraciones de mayor dominio sobre ciertos temas y mayor capacidad para llevar a cabo tareas pesadas.

Al igual que los estudios de Lupton (2000), quien señala temor de los hombres a ser feminizados y estigmatizados como afeminados y homosexuales, en el presente estudio se presentan aseveraciones de los entrevistados, en términos de que la convivencia con las mujeres hace que algunos de sus compañeros en dichas profesiones se conviertan en "afeminados" $u$ "homosexuales".

Evans (2002) y Taylor (2012) señalan que los varones en profesiones feminizadas evitan ciertas tareas por el temor de que sean malinterpretadas como abuso sexual. En particular, Evans (2002) señala que, el contacto físico con los pacientes o estudiantes por parte del hombre es visto con suspicacia por su posible naturaleza sexual. Los entrevistados en este estudio reportaron cuestiones similares, se encontró que, ellos evitan el contacto con las menores y para hablar de temas de sexualidad, la relación del varón con las menores (estudiantes) debe de estar mediada por una mujer.

En este estudio, en particular los enfermeros señalaron trato diferenciado por parte de los pacientes hacia los enfermeros y enfermeras. Algunos pacientes, como lo es el caso de las mujeres adultas mayores, exigen ser atendidas por una enfermera. Este trato desigual fue identificado también en el estudio de Das, Singh, Pawar y Guha (2012), quienes señalan haber encontrado incomodidad por parte de los enfermeros para ejercer su profesión, lo que se asocia al trato desigual recibido por parte de los pacientes. 
Por último, en el tercer eje de análisis, sobre las prácticas de cuidado fuera de la profesión, a través del presente estudio se encontró que los hombres en profesiones feminizadas, cuidan de sus hijos en el hogar, incluso a pesar de estar divorciados o separados de las madres de sus hijos. Este último elemento coincide con el estudio de Aguayo y Sadler (2012), quienes señalan que los varones a pesar de la separación de sus parejas siguen procurando el cuidado de sus hijos, en buena medida si se asocia a una práctica que se ha normalizado como parte de su cotidianidad.

\section{ÚLTIMAS CONSIDERACIONES}

Los varones en profesiones feminizadas se enfrentan a diferentes tensiones para ejercer su profesión y para el desarrollo de su vida familiar. Las problemáticas que fueron enfatizadas incluyen la presión familiar para cambiar a una profesión más "varonil" o "redituable"; miedo a ser acusado de abuso sexual por contacto físico, que es señalado por ellos como de "naturaleza no sexual"; discriminación de los pacientes hacia los enfermeros por ser hombres; demanda de un rol autoritario en el espacio laboral y, discriminación laboral en hospitales por ser hombre. Estos hombres también advirtieron ventajas dentro de su profesión, entre estas: la satisfacción de cuidar y enseñar, el convivir con mujeres, y la adrenalina asociada a su trabajo. Los varones también reportaron satisfacción en el involucramiento de las actividades de cuidado en el hogar.

No se busca victimizar ni idealizar su presencia en labores de cuidado, ni limitarnos a destacar coincidencias con otros estudios hechos en contextos nacionales distintos, sino de enfatizar la pertinencia de documentar y buscar formas de nombrarlo, con el fin de estimular procesos reflexivos y de investigación sobre estrategias individuales o grupales de acomodación, resistencia y transgresión a normatividades y aprendizajes de género. Existen algunas áreas que restarían por ser profundizadas, como la percepción de la naturaleza del contacto físico, asociado a las construcciones sociales de los cuerpos. Otro de los temas son las barreras institucionales a las que se enfrentan los varones con una doble tensión. Por un lado, los varones se enfrentan con límites al ejercer su profesión, es decir, el medio les impone barreras como lo son: el no hablar de temas de sexualidad, o el no poder atender a pacientes mujeres. Por el otro lado, el medio les demanda reafirmar su rol de autoridad como varones, de quienes se espera fuerza física, y carácter. Aunque ellos no quieran verse inmersos en ambas tensiones, el medio les limita, y les exige. Este elemento, demanda profundizar sobre las paredes de cristal (por analogía con los denominados techos de cristal) 
a las que también se enfrentan los varones en los espacios de cuidado tradicionalmente feminizados.

\section{REFERENCIAS BIBLIOGRÁFICAS}

Agadjanian, V., 2002, “Men doing “women's work": Masculinity and gender relations among street vendors in Maputo, Mozambique". The Journal of Men's Studies, 10(3), 329-342.

Aguayo, F. y Sadler, M., 2012, "Men who care: Chile. A qualitative study on men and caregiving", en Barker, G., Greene, M., Nascimento, M., Segundo, M., Ricardo, C., Taylor, A., Aguayo, F., Sadler, M., Das, A., Singh, S., Figueroa, J. G., Franzoni, J., Flores, N., Jewkes, R., Morrell, R. y Kato, J., Men who care: a multi-country qualitative study of men in non-traditional caregiving roles. Washington, D.C.: International Center for Research on Women (ICRW) y Rio de Janeiro: Instituto Promundo.

Arango, Luz Gabriela y Molinier, Pascale, 2011, El trabajo y la ética del cuidado. La Carreta Social. Universidad Nacional de Colombia.

Barker, G., Greene, M., Nascimento, M., Segundo, M., Ricardo, C., Taylor, A., Aguayo, F., Sadler, M., Das, A., Singh, S., Figueroa, J. G., Franzoni, J., Flores, N., Jewkes, R., Morrell, R. y Kato, J., 2012, Men who care: a multi-country qualitative study of men in non-traditional caregiving roles. Washington, D.C., International Center for Research on Women (ICRW) y Rio de Janeiro: Instituto Promundo.

Bourdieu, P., 2000, La dominación masculina. Barcelona, Anagrama.

Buquet, A., 2006, Presencia de hombres y mujeres en la UNAM: una radiografía. Programa Universitario de Estudios de Género, Universidad Nacional Autónoma de México.

Connell, R., 2015, Masculinidades. Programa Universitario de Estudios de Género, Universidad Nacional Autónoma de México.

Das, A., Singh, S. Pawar, A y Guha, M., 2012, "Men who Care: Brazil. A study of men in caregiving and alternative roles and professions in India", en Barker et al., Men who care: a multi-country qualitative study of men in non-traditional caregiving roles, Washington, D.C.: International Center for Research on Women (ICRW) y Rio de Janeiro: Instituto Promundo.

Evans, J., 2002, "Cautious caregivers: Gendered stereotypes and the sexualization of men nurses' touch”. Journal of Advanced Nursing, 40(4), 441-448.

Evans, J., 1997, "Men in nursing: issues of gender segregation and hidden advantage". Journal of advanced nursing, 26(2), 226-231.

Evans, J., y Frank, B., 2003, "Contradictions and tensions: Exploring relations of masculinities in the numerically female-dominated nursing profession". The Journal of Men's Studies, 11(3), 277-292. 
Figueroa, J.G., 2018, "Salud y mortalidad paternas desde el cuidado de sí y de otros: algunas reflexiones", El trabajo de cuidados: una cuestión de derechos humanos y políticas públicas. ONU Mujeres, pp. 124-138.

Figueroa, J. G. y Flores, N., 2012, "Prácticas de cuidado y modelos emergentes en las relaciones de género. La experiencia de algunos varones mexicanos”. Revista de Estudios de Género. La ventana, 4(35), 7-57.

Figueroa, J. G. y Franzoni, J., 2011, "Del hombre proveedor al hombre emocional: construyendo nuevos significados de la masculinidad entre varones mexicanos", en Aguayo, F. y Sadler, M. (eds.) Masculinidades y Politicas Púbicas. Involucrando hombres en la equidad de género (pp. 64-81). Chile: Universidad de Chile.

Figueroa, J.G., L. Jiménez y O. Tena, 2006, Padres, esposos e hijos. Prácticas y valoraciones de varones mexicanos. El Colegio de México.

Flyvbjerg, B., 2006, "The power of example (Cases and 'casing')", en Making Social Science Matter. Cambridge University Press, pp. 76-87.

Greenberg, E., y Levine, B., 1971, "Role strain in men nurses. A preliminary investigation". Nursing Forum, 10(4), 416-430.

Heikes, E. J., 1991, "When men are the minority: The case of men in nursing". The Sociological Quarterly, 32 (3), 389-401.

INEGI, 2015, Encuesta Nacional de Ocupación y Empleo (ENOE) 2015, México: Instituto Nacional de Geografía y Estadística (INEGI).

INEGI, 2014, Encuesta Nacional de Ocupación y Empleo (ENOE) 2014, México: INEGI.

Jiménez, L., 2003, Dando voz a los varones. Sexualidad, reproducción y paternidad de algunos mexicanos. México, UNAM/CRIM.

Kleinman, C. S., 2004, “Understanding and capitalizing on men's advantages in nursing". Journal of Nursing Administration, 34(2), 78-82.

Lara, V., 2012, Masculinidades en el trabajo. Lógicas de acción y definiciones subjetivas de varones enfermeros, (Tesis doctoral). El Colegio de México: Ciudad de México.

Lupton, B., 2006, “Explaining men's entry into Female Concentrated occupations: issues of masculinity and social class". Gender, Work and Organization, 13(2), 103-128.

Lupton, B., 2000, "Maintaining masculinity: men who do 'women's work"'. British Journal of Management, 11(s1), 33-48.

Meadus, R. J., 2000, "Men in nursing: Barriers to recruitment". Nursing Forum, 35(3), 5-12.

Okrainec, G., 1994, "Perceptions of nursing education held by male nursing students". Western Journal of Nursing Research, 16(1), 94-107. 
Molinier, Pascale, 2011, “Antes que todo, el cuidado es un trabajo", en Arango, Luz Gabriela y Pascale Molinier (compiladoras), El trabajo y la ética del cuidado. La Carreta Social. Universidad Nacional de Colombia, pp. 45-64.

Perujo, E., 2015, ¿Qué clase de paternidad es esa? El trabajo de parentesco desde la incertidumbre. Tesis de doctorado en Antropología. Universidad nacional Autónoma de México.

Petchesky, R. y K. Judd, 1998, Negotiating reproductive rights. Women's perspectives across countries and cultures, Zed Books, New York, E.U.

Piper, P. S., y Collamer, B. E., 2001, "Male librarians: Men in a feminized profession". The Journal of Academic Librarianship, 27(5), 406-411.

Romem, P., y Anson, O., 2005, "Israeli men in nursing: social and personal motives". Journal of Nursing Management, 13(2), 173-178.

Schwalbe, M. y Wolkomir, M., 2001, "The masculine self as problem and resource in interview studies of men", Men and Masculinities, vol. 4, núm. 1, pp. 90-103.

Simpson, R., 2004, "Masculinity at work the experiences of men in female dominated occupations". Work, Employment and Society, 18(2), 349-368.

Taylor, A., 2012, "Men who care: Brazil. a case study of men engaging in care roles", en Barker et al., Men who care: a multi-country qualitative study of men in non-traditional caregiving roles. Washington, D.C.: International Center for Research on Women (ICRW) y Rio de Janeiro: Instituto Promundo.

Tronto, J., 1993, Moral Boundaries, a political argument for an ethic of care. Routledge, USA.

Villeneuve, M., 1994, "Recruiting and retaining men in nursing: A review of the literature". Journal of Professional Nursing, 10 (4), 217-228.

Williams, C. L., 1995a, Still a man's world: Men who do women's work (vol. 1). Berkeley y Los Angeles, California: University of California Press.

Williams, C. L., 1995b, "Hidden advantages for men in nursing”, Nursing Administration Quarterly, 19(2), 63-70.

Williams, C. L., 1992, "The glass escalator: hidden advantages for Men in the 'female' professions". Social Problems, 39(3), 253-267. 


\section{RESUMEN CURRICULAR DEL AUTOR}

\section{Juan Guillermo Figueroa Perea}

Profesor e investigador de El Colegio de México y profesor de asignatura en la Universidad Nacional Autónoma de México. Investiga sobre comportamientos reproductivos de los varones, sobre ética de la investigación social, así como sobre derechos reproductivos y sexuales en su relación con políticas públicas y con los discursos religiosos. Forma parte del Sistema Nacional de Investigadores en el nivel III. Es editor de diez libros sobre sexualidad, reproducción y salud, la mayoría publicados en El Colegio de México y en la UNAM.

Dirección electrónica: jfigue@colmex.mx

Registro ORCID: http://orcid.org/0000-0002-2918-8145

\section{Tania Hernández}

Licenciada en Sociología y Maestra en Estudios Políticos y Sociales por la Universidad Nacional Autónoma de México. 